\title{
Opportunities And Challenges-The Next 5 Years of a Service Dedicated to the Medical Management of Neurosurgical Patients
}

\author{
Rene Daniel ${ }^{1,2, *} ;$ Catriona McDonald Harrop ${ }^{1,2}$ \\ ${ }^{1}$ Farber Hospitalist Service, Department of Neurological Surgery, Thomas Jefferson \\ University, Philadelphia, PA 19107 \\ 2Department of Medicine, Thomas Jefferson University, Philadelphia, PA 19107
}

*Corresponding author

Keywords: interactions, education, research, improvement, patient care, publishing

\begin{abstract}
Neurosurgery hospitalists will continue to be closely involved in the management of patients which undergo neurosurgery. In the first article, we discuss the tremendous potential of the service for improving the care for neurosurgical patients at Jefferson. We highlight the interactions with other services and the areas for potential growth. Further, we emphasize the need for constant feedback, which is necessary to improve the care provided by FHS.

In the second part of the article, we highlight the potential impact of FHS beyond the Jefferson Hospitals in Center City of Philadelphia, to other hospitals within the Jefferson network, and beyond Jefferson. We re-emphasize the educational opportunities FHS is able to provide for other neurosurgery hospitalists nationwide and on international level, and the future research and education program.
\end{abstract}

We hope that this article will increase understanding of other physicians of FHS work, and that it will open new interactions with other services both at Jefferson and beyond.

\section{MANUSCRIPT}

Over last 5 years, FHS became closely integrated part of the Department of Neurological Surgery, proving both preoperative and postoperative care for neurosurgical patients, as well as orthopedic spine patients of the Department of Orthopedics. In addition to consulting services, FHS also serves as the primary team for many neurosurgical patients, particularly those with multiple medical comorbidities. FHS role as the primary team is appreciated by other services, as reflected in increasing preferences for patients to be placed on the FHS service. In taking care of neurosurgical patients as a primary team, FHS helps surgical teams to increase the number of procedures performed at Jefferson. Recently, FHS was also tasked by Jefferson with providing consultative and co-management care for the ophthalmology services at the Wills Eye Hospital and the Jefferson Hospital of Neuroscience, as the need for the hospitalist support for ophthalmology became apparent.

The increasing demand placed on the service is appreciated, but at the same time strains the service resources. This is due to the above stated fact - FHS is an attendingrun service, which until very recently did not utilize NPs or residents. Thus, patient care, from admission to discharge, is provided by the attending physician only, although with the support from other consulting services, which is of course highly appreciated. FHS is working to address the situation on multiple ways, with the objective to deliver the highest possible patient care: First, FHS is working closely with other services which admit similar patients, i.e. neurosurgery, orthopedic spine, general medicine and trauma as well as the Emergency Department, to coordinate admission criteria in order to place patients onto appropriate services, and to avoid inappropriate admissions. A continuous feedback from neurosurgeons is necessary. Second, FHS was pleased to welcome this year its first nurse practitioner (NP). Greater Involvement of NPs is expected to help with appropriate management of resources and will ultimately lead to increased number of patients accepted by FHS. Third, close interactions with the nursing staff is crucial to the FHS success. Thus, training and education of the nursing staff by FHS will become an important aspect of the FHS work (more on that below).

After establishment of the FHS, other Jefferson departments, following its example, established their own services. These include Medical Oncology and Gastroenterology, both who now have dedicated Hospitalist services. FHS is always willing to share the practical experience and help in establishment of this care model, in order to ensure high quality patient care.

In the Jefferson network outside of the Center City, there is no FHS-like dedicated service. Likewise, hospitalist services for neurosurgeons around the country and internationally are usually provided at a consulting or co-management level, and dedicated primary services like FHS to our knowledge do not exist. The situation is aggravated by a scarcity of guidelines how to manage medical problems in neurosurgical patients. Given the overall positive experience with FHS at Jefferson, it would seem reasonable to initiate cooperation and collaborations with other hospitals at Jefferson and beyond with the objective to standardize patient care and decrease inefficiencies, thus decreasing length of stay, mortality, complications and readmissions. Indeed, FHS took the fist step by producing, together with neurosurgery and many other services of Jefferson, the first textbook in the field. ${ }^{1}$ We hope this text will become a standard of care at Jefferson and beyond when it comes to medical management of these patients. 
Table 1. Mortality of patients with epidural abscess at Jefferson.

Mortaility, FHS vs. Other Services

\begin{tabular}{|l|c|c|c|c|}
\hline & N total cases & N total cases & Overall Mortality \\
\hline FHS & 48 & 0 & $0.0 \%$ & $p=0.026$ \\
\hline Other & 48 & 6 & $12.5 \%$ & \\
\hline
\end{tabular}

In summary, the previous paragraph outlines a set of challenges and opportunities for neurosurgery hospitalists both at Jefferson and nationwide. The standardization of guidelines and patient care across multiple hospitals is paramount. The published textbook is only the first step in this direction and needs to be build upon. In addition to increasing awareness of the book by presentations at national and international meetings, the textbook should be updated in a timely manner, and a second edition may be needed in not-so-distant future. Involvement of non-Jefferson physicians in its creation may be also possible. In addition, other educational opportunities arise on the heels of the book. At present, there is no symposium which is dedicated to the education of neurosurgery hospitalists. Organization of this type of event at Jefferson would improve education and confirm the leading role of FHS in this process. Finally, education does not stop at once a year event and a book. An attractive option currently being explored is the creation of an online course for neurosurgery hospitalists, which could be accessed worldwide. FHS, together with other Jefferson departments have the resources to perform this highly useful service and create new education opportunities worldwide. Last but not least, educational resources should be present "at one's fingertips". Following this rule, FHS created its own smart phone application, which address the questions which arise from day to day work, from preoperative risk stratification to admissions to treatment protocols. Further improvement of the application and validation of thereof should again lead to improvement in patient care.

Patient care always brings to light many areas where clinicians run into the limits of human knowledge. It is the duty of any academic service to increase our understanding of the human body, its diseases and the diagnostic and treatment options available. Generally, there are two approaches to do so. First, it is always desirable to evaluate retrospectively the failures and processes in patient management. FHS here engages statisticians and is in the process of evaluating the impact of FHS physicians on the patient care, starting with mortality, length of stay and readmissions. Interestingly, our early data suggest that the mortality of patients with epidural abscess is strikingly lower when under care of FHS as a primary team, when compared to other services (Table 1). The statistical analysis is ongoing, and it is hoped it will point for us areas of potential improvement, which can be addressed.

Second, new, prospective research studies can address new hypothesizes, which were created on the back of newly available technologies and knowledge. Thus, new clinical studies in preoperative care, currently also ongoing, and collaboration with other services such as infectious diseases will keep FHS at the cutting edge of the field. At the same time, it is crucial to exchange experiences and results with other similar services nationwide. Thus, FHS will work toward collaboration with other similar services across the country. Incorporation of new approaches from other services should be implemented efficiently, such as, for example, improvement of preoperative risk stratification based on the Canadian guidelines. $^{2}$

Taken together, neurosurgery hospitalist work in general and FHS work in particular is a continuously changing process, which is influenced by interactions with with and requests for help from other services, as well as by new data, either coming in from its own research or from other physicians' studies. However, the strive for the highest quality patient care remains constant.

\section{REFERENCES}

1. aOUPAcademic. Medical Management of Neurosurgical Patients. aOUPAcademic; 2020.

2. Canadian Cardiovascular Society Guidelines on Perioperative Cardiac Risk Assessment and Management for Patients Who Undergo Noncardiac Surgery - Canadian Journal of Cardiology. 2020. 